(C) 2006 The Japan Society of Applied Physics

\title{
Single-Run Single-Mask Inductively-Coupled-Plasma Reactive-Ion-Etching Process for Fabricating Suspended High-Aspect-Ratio Microstructures
}

\author{
Yao-Joe YANG*, Wen-Cheng KUO and Kuang-Chao FAN \\ Department of Mechanical Engineering, National Taiwan University, No. 1 Roosevelt Rd., Sec. 4, Taipei, Taiwan, R.O.C.
}

(Received June 13, 2005; accepted September 7, 2005; published online January 10, 2006)

In this work, we present a single-run single-mask (SRM) process for fabricating suspended high-aspect-ratio structures on standard silicon wafers using an inductively coupled plasma-reactive ion etching (ICP-RIE) etcher. This process eliminates extra fabrication steps which are required for structure release after trench etching. Released microstructures with $120 \mu \mathrm{m}$ thickness are obtained by this process. The corresponding maximum aspect ratio of the trench is 28 . The SRM process is an extended version of the standard process proposed by BOSCH GmbH (BOSCH process). The first step of the SRM process is a standard BOSCH process for trench etching, then a polymer layer is deposited on trench sidewalls as a protective layer for the subsequent structure-releasing step. The structure is released by dry isotropic etching after the polymer layer on the trench floor is removed. All the steps can be integrated into a single-run ICP process. Also, only one mask is required. Therefore, the process complexity and fabrication cost can be effectively reduced. Discussions on each SRM step and considerations for avoiding undesired etching of the silicon structures during the release process are also presented.

[DOI: $10.1143 / \mathrm{JJAP} .45 .305]$

KEYWORDS: inductively coupled plasma (ICP), high-aspect-ratio-structure (HARS), bulk micromachining, deep reactive ion etching (DRIE), suspended microstructures

\section{Introduction}

High-aspect-ratio structures (HARS) are widely used in micro electro mechanical systems (MEMS), particularly in devices with suspended movable structures that are designed for in-plane motions. Such devices include micro-gyroscopes, accelerometers, optical mirrors, and optical shutters. The major advantage of HARS over structures fabricated by the surface-micromachining process is that suspended HARS are relatively stiff in the out-of-plane direction, but compliant in the in-plane direction. The fabrication of HARS requires etching technologies with the following characteristics: (1) etchers with a high etch rate, (2) etchants with good selectivity to the mask material, (3) high anisotropy, and (4) compatibility with other processes. ${ }^{1)}$ Conventional technologies, such as reactive ion etching (RIE) and electron cyclotron resonance (ECR), permit an anisotropic profile. However, their application to HARS fabrication is limited by the fact that their etching rates as well as the selectivity to mask materials (e.g., silicon dioxide) are relatively low. ${ }^{2)}$

For silicon etching with a high etching rate and high selectivity, fluorine-based etchant is the common choice. The main advantage of fluorine-based etchant is its high etching rate of silicon. However, for fabricating HARS, its major drawback is the characteristic of isotropic etching. In order to maintain high anisotropic profiles of etched structures, the technique of sidewall passivation during etching has been proposed. ${ }^{3)}$ The process using inductively coupled plasma (ICP) sources with the process proposed by $\mathrm{BOSCH} \mathrm{GmbH}$ (BOSCH process) ${ }^{4)}$ can effectively balance the steps between etching and passivation, satisfying the requirement of fabricating HARS, and therefore is widely used for HARS trench etching. Most of the studies on ICP etching are focused on the characteristics of trench etching, such as profile control for high-aspect-ratio etching, ${ }^{5}$ optimum etching rate, ${ }^{6}$ uniformity improvement, ${ }^{7)}$ the

\footnotetext{
*Author to whom correspondence should be addressed.

E-mail address: yjy@ntu.edu.tw
}

reduction of the notch effect, ${ }^{8)}$ and the reduction of sidewall roughness. ${ }^{9)}$

For creating a suspended HARS, a structure-releasing step is required after trench etching. There are many approaches to structure release. These approaches include single-crystal reactive etching and metallization (SCREAM), ${ }^{10)}$ the black silicon method (BSM), ${ }^{11)}$ the silicon-on-insulator (SOI) process, ${ }^{12)}$ surface/bulk micromachining (SBM), ${ }^{13)}$ the dissolved wafer process (DWP) ${ }^{14)}$ the boron etch-stop assisted lateral silicon etching (BELST) process, ${ }^{15)}$ the aluminum interconnect process for air-gap-insulated microstructures (AIM) ${ }^{16)}$ and the process proposed by Sarajlic et al. ${ }^{17)}$ The SOI wafer process involves the use of the buried oxide layer as the sacrificial layer for wet release. This process is quite straightforward for structure release and is easy to control. However, the wafer cost is about two orders higher than that of standard silicon wafers. The suspended structures released by the DWP process have very high dopant concentrations so that they usually have inferior mechanical properties. For the processes proposed in refs. 10, 11, 13, 15, 16, and 17, extra fabrication steps, such as plasma-enhanced chemical vapor deposition (PECVD), ion implantation, or sputtering, are required for structure release after trench etching.

In this paper, we propose a single-run single-mask (SRM) process for fabricating a suspended HARS using an ICP-RIE etcher. In the proposed process, the standard $\mathrm{BOSCH}$ process is used for trench etching. After the trench is etched to a desired depth, the sidewall protective layer is created by a polymer deposition step that is similar to the passivation step of the BOSCH process. The relationship between the polymer deposition rate and trench aspect ratio/trench depth is also discussed. Then, a polymer-removing step, which is similar to the etching step of the standard BOSCH process, is used to remove the polymer layer at the bottom of trenches. The mechanisms and the characteristics of floor polymer removal times/rates under different process conditions are provided. Finally, the structure is released by the etching step of the BOSCH process without platen power. The relationship between lateral etching rate and trench opening 


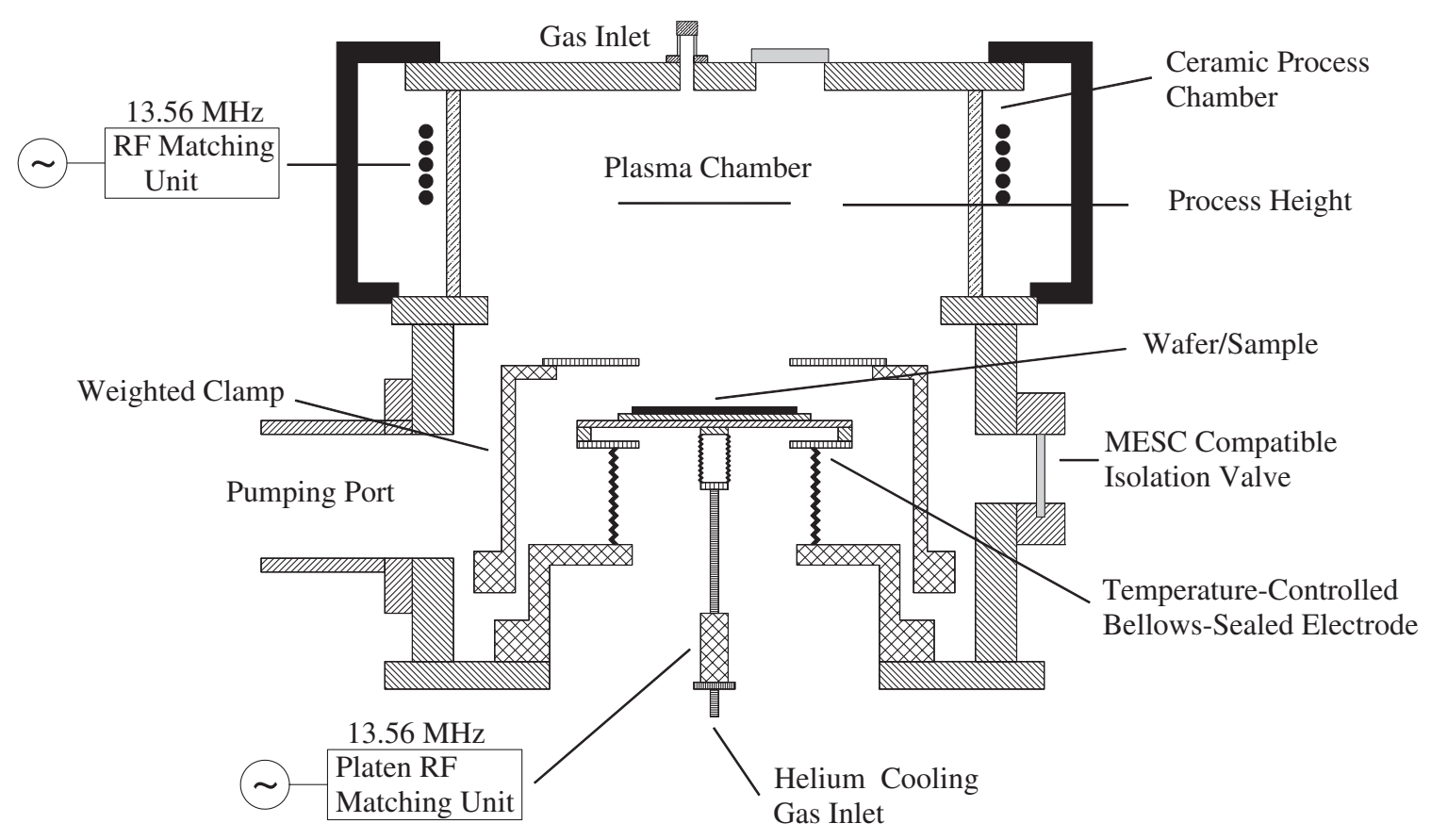

Fig. 1. Schematic of STS ICP-RIE system.

area/trench depth is also studied. All the steps can be integrated into a single-run ICP process so that the process complexity and fabrication cost can be effectively reduced.

This paper is organized as follows: the background on the $\mathrm{BOSCH}$ process is described in $\$ 2$. The SRM process for fabricating released structures in a single-run ICP-RIE process with five steps is described in $\S 3$. In $\S 4$, we will discuss the challenges and the important factors of the fabrication process. Our conclusions are presented in $\$ 5$.

\section{Background on the BOSCH Process}

A high-density ICP etcher, which is the major process equipment used in this work, is illustrated in Fig. 1. The plasma is created within the plasma chamber using an inductive source coil supplied by a $13.56 \mathrm{MHz}$ RF generator. Another $13.56 \mathrm{MHz}$ RF generator, which is connected to the platen, is used to create a bias between the plasma and the workpiece. ${ }^{18)}$ Fig. 2 shows the operating principle of the BOSCH process. ${ }^{4)}$ The first step, as shown in Fig. 2(a), is a single short-time isotropic etching of silicon on a patterned substrate using fluorine-containing plasma. Second, the etched trench is passivated by depositing a Teflon-like polymer as a protective layer, as shown in Fig. 2(b). In the next step, the polymer layer on the bottom is removed by ion bombardment, so that the etchant continues to react with the exposed silicon, as shown in Fig. 2(c). On the other hand, lateral etching is avoided since the sidewall polymer layer still remains after the ion bombardment. Again, a passivation follows the etching step, as shown in Fig. 2(d). Note that the etching and deposition times are usually less than $10 \mathrm{~s}$. The $\mathrm{BOSCH}$ process is in fact a segregation of etching and passivation steps which enable deep trench etching, and can be used to fabricate high-aspect-ratio MEMS structures.

\section{SRM Process for Fabricating Released Structures}

As described in the previous section, the $\mathrm{BOSCH}$ process is a widely used tool for fabricating high-aspect-ratio

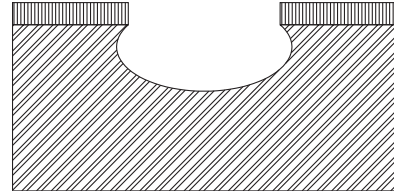

(a) etching cycle

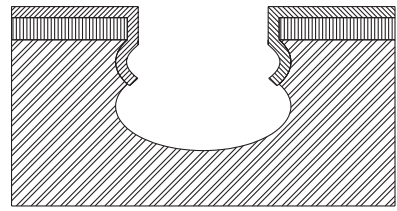

(c) etching cycle

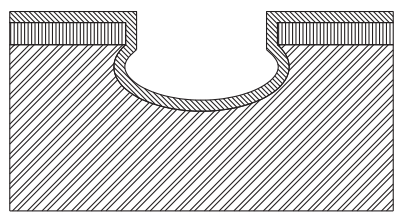

(b) passivation cycle

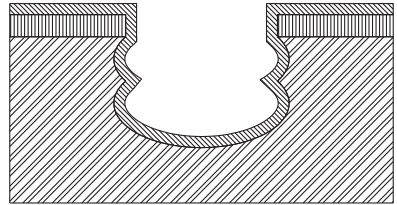

(d) passivation cycle

\section{$\mathbb{Z}$ silicon substrate $\quad \mathbb{D}$ PR $\quad \mathbb{N}$ polymer}

Fig. 2. Schematic of the principle of the $\mathrm{BOSCH}$ process.

trenches on single-crystal silicon wafers. Making use of the characteristics of the etching and passivation steps of the $\mathrm{BOSCH}$ process, we design a new process for fabricating high-aspect-ratio suspended MEMS structures in a singlerun ICP process. The steps of this single-run process are illustrated in Fig. 3. This process includes five steps, and the detailed descriptions of these steps are as follows

Pre-SRM step: Patterning of silicon substrate

Before the ICP process, photoresist is spun onto a silicon wafer as the etching mask for deep silicon etching, followed by a pattern transfer process.

Step 1: Trench etching

This step is in fact a standard BOSCH process. The exposed silicon is then etched to the desired depth.

Step 2: Polymer deposition

This step is similar to the passivation step of the standard $\mathrm{BOSCH}$ process, except that the time for depositing the passivation layer is about $10-25 \mathrm{~min}$. 


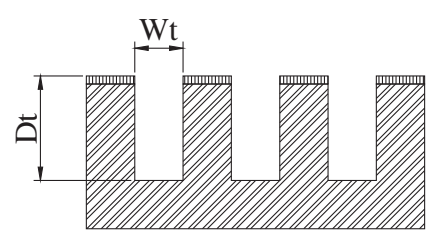

1. Trench etching

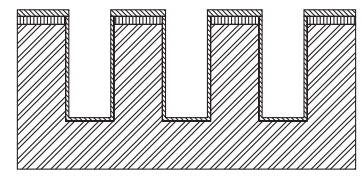

2. Polymer deposition

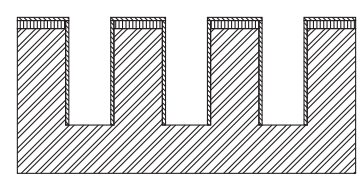

3. Floor polymer removal

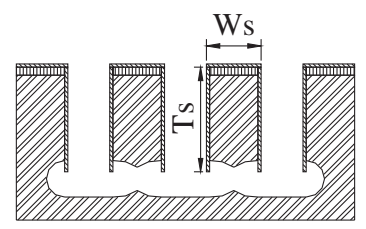

4. Structure release

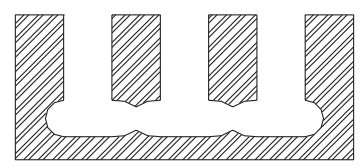

5. PR and polymer stripping

\section{Vilicon}

Fig. 3. Process flow of the SRM process.

The purpose of this step is to deposit a fluorocarbon polymer layer around the structure surface so that the sidewalls of trenches will not be etched during the following release step.

Step 3: Floor polymer removal

This step is similar to the etching step of the standard $\mathrm{BOSCH}$ process, except that the time for floor polymer removal is about $10-20 \mathrm{~min}$. The etchant, which consists of fluorine radicals, is used to remove the floor polymer with the assistance of ion bombardment.

Step 4: Structure releasing
In this step, the exposed silicon is isotropically etched by using the BOSCH etching step without any platen power. The purpose of this step is to release structures.

Step 5: PR and polymer stripping

In this step, oxygen plasma is used to strip the photoresist and polymer.

Post-SRM Step: Conduction layer deposition

- When the depth/width ratio of a trench is less than 5, an insulation layer for circuit isolation is deposited by using the passivation step of the standard BOSCH process to form a polymer layer. The procedure is the same as that of Step 2. Then an aluminum-sputtering process can be adopted to create a conductive layer around the released microstructure. This step is similar to the metallization step of the SCREAM process. ${ }^{10)}$

- When the depth/width ratio of a trench is larger than 5 , a metal sputtering process will not be able to give a uniformly deposited layer on the sidewalls of microstructures. Therefore, the method proposed in ref. 19 can be applied to create a uniform conductive layer on the structure.

Table I lists typical process parameters used in the SRM process. (Further discussion of this table will be provided in the next section.)

\section{Process Results and Discussion}

In $\S 3$, we proposed the SRM process, which employs the etching and passivation steps of the standard BOSCH process to fabricate a released microstructure in a singlerun ICP process. It is obvious that, except for the first step (the standard $\mathrm{BOSCH}$ process for trench etching), all the steps are variations of the passivation and etching steps of the standard BOSCH process. In this section, we will present the process results and the discussion of the SRM process steps.

\subsection{Examples of fabricated structures}

Figure 4 shows the cross section of comb structures with $120 \mu \mathrm{m}$ thickness fabricated by the SRM process. The width of each comb finger is $10 \mu \mathrm{m}$ and the minimum gap between fingers is $5 \mu \mathrm{m}$. The maximum aspect ratio of the structure is 12 , and the maximum aspect ratio of the trench is 28 . Note that here we define the aspect ratio of the structure $\left(A R_{\mathrm{S}}\right)$ as

$$
A R_{\mathrm{S}}=\frac{T_{\mathrm{S}}}{W_{\mathrm{S}}}
$$

and the aspect ratio of the trench $\left(A R_{\mathrm{T}}\right)$ as

Table I. Polymer removal time for different process parameters based on 25-min polymer deposition with a source power of $600 \mathrm{~W}$, a $\mathrm{C}_{4} \mathrm{~F}_{8}$ flow rate of $99 \mathrm{sccm}$, and an APC angle of $81.2^{\circ}$. The aspect ratio for this experiment is 3.5 .

\begin{tabular}{|c|c|c|c|c|c|c|c|}
\hline Parameter no. & 1 & 2 & 3 & 4 & 5 & 6 & 7 \\
\hline Coil power (W) & 800 & 600 & 600 & 600 & 600 & 600 & 600 \\
\hline $\mathrm{SF}_{6} / \mathrm{O}_{2}(\mathrm{sccm})$ & $150 / 13$ & $150 / 13$ & $130 / 13$ & $130 / 13$ & $130 / 13$ & $130 / 13$ & $130 / 13$ \\
\hline APC angle (deg) & 50 & 50 & 50 & 65 & 81.2 & 65 & 81.2 \\
\hline $\begin{array}{l}\text { Floor polymer } \\
\text { removal time }(\mathrm{min})\end{array}$ & 10.5 & 11 & 11.5 & 12 & 16.5 & 14.5 & 18 \\
\hline $\begin{array}{l}\text { Polymer removal } \\
\text { rate }(\mu \mathrm{m} / \mathrm{min})\end{array}$ & 0.238 & 0.227 & 0.217 & 0.208 & 0.152 & 0.172 & 0.139 \\
\hline
\end{tabular}




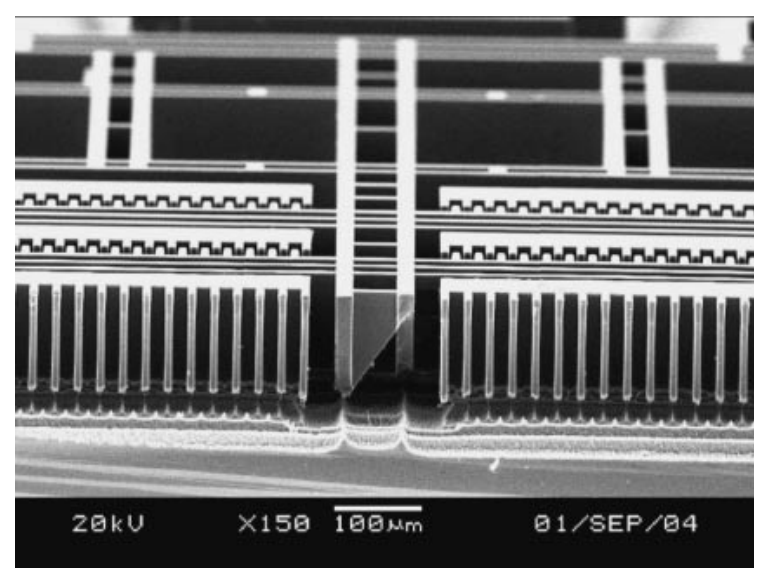

Fig. 4. Cross section of a comb-drive array structure with a thickness of $120 \mu \mathrm{m}$ fabricated by the SRM process.

$$
A R_{\mathrm{T}}=\frac{D_{\mathrm{T}}}{W_{\mathrm{T}}}
$$

where $T_{\mathrm{S}}$ and $W_{\mathrm{S}}$ are the thickness and width of the structure, respectively, and $D_{\mathrm{T}}$ and $W_{\mathrm{T}}$ are the depth and width of the trench, respectively. These variables are schematically shown in Fig. 3.

\subsection{Sufficient polymer deposition time}

In the SRM process, the deposited polymer layer is used to protect against sidewall erosion during Steps 3 and 4. Due to the conductance effect $^{20}$ ) and the reactant transport effect, ${ }^{21)}$ the flux distribution of CF-based species around a trench profile is affected by the aspect ratio of the trench. The deposition rate at the trench bottom is usually smaller than that on top of the trench. Figure 5 shows the relationship between the polymer deposition rate and the trench aspect ratio (or trench depth). Note that the conditions for depositing the polymer are as follows: the source coil power

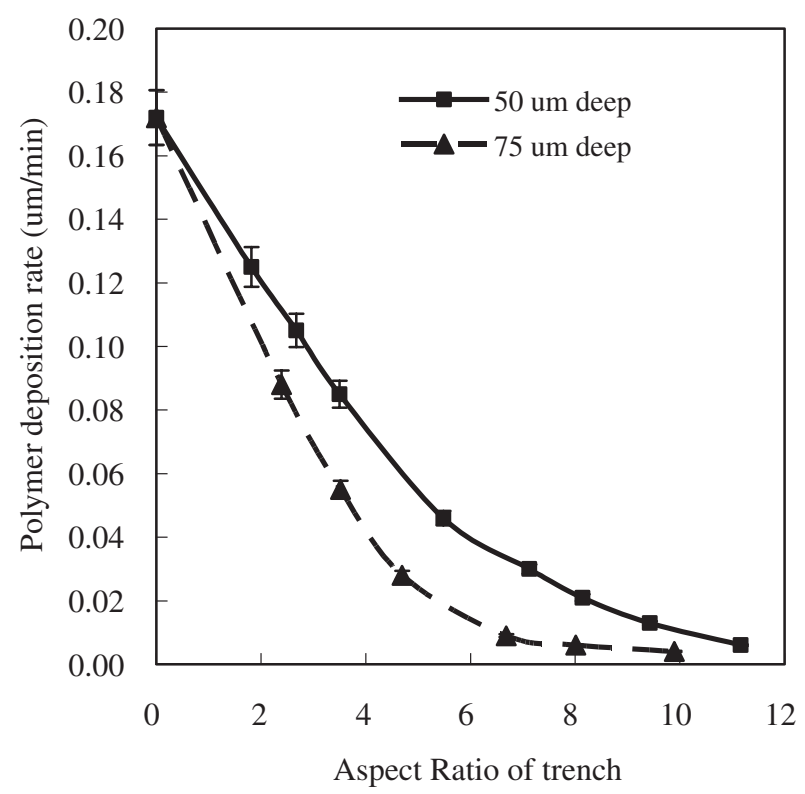

Fig. 5. Polymer deposition rates on trench bottom vs aspect ratio of trench. The conditions for depositing the polymer were as follows: the source coil power was $800 \mathrm{~W}$, the $\mathrm{C}_{4} \mathrm{~F}_{8}$ flow rate was $99 \mathrm{sccm}$, the APC angle was $81.2^{\circ}$, and the deposition time was $25 \mathrm{~min}$.

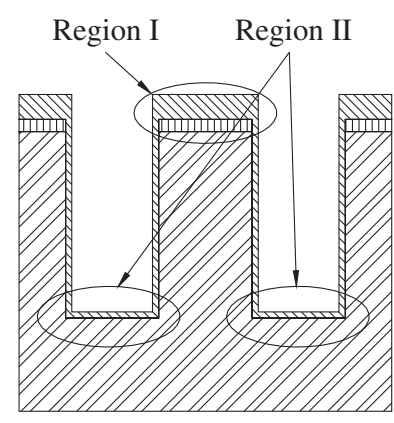

(a)

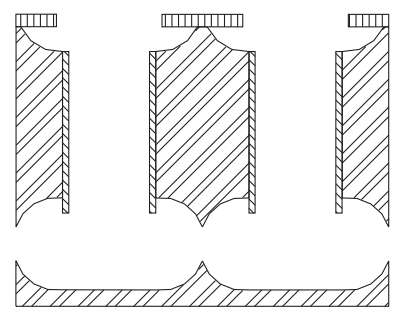

(c)

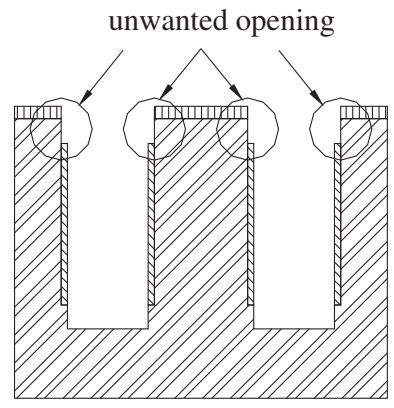

(b)

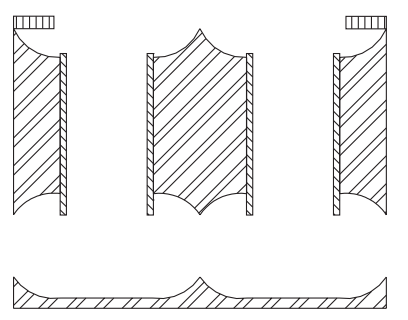

(d)

\section{$\square$ Silicon $\quad$ |lा PR P Polymer}

Fig. 6. (a) Polymer on top of the trench (Region I) is thicker than that on the bottom of the trench (Region II). (b) An "unwanted opening" is formed if the etching time for bottom polymer removal is too long. (c) Undesired etching of silicon structures through the unwanted openings during the release process. (d) After photoresist removal.

Table II. Measured polymer sidewall thicknesses [see Fig. 6(a)] for two different structure thicknesses. These results were obtained with 25-min polymer deposition with a source power of $800 \mathrm{~W}$, a $\mathrm{C}_{4} \mathrm{~F}_{8}$ flow rate of $99 \mathrm{sccm}$, and an APC angle of $81.2^{\circ}$.

\begin{tabular}{cccc}
\hline \multirow{2}{*}{$\begin{array}{l}\text { Structure } \\
\text { thickness }(\mu \mathrm{m})\end{array}$} & $\begin{array}{c}\text { Trench aspect } \\
\text { ratio }\end{array}$ & \multicolumn{2}{c}{ Polymer sidewall thickness $(\mu \mathrm{m})$} \\
\cline { 3 - 4 } & 10 & Region I & Region II \\
\hline 50 & 10 & 0.8 & 0.19 \\
75 & 1.3 & 0.12 \\
\hline
\end{tabular}

is $800 \mathrm{~W}$, the $\mathrm{C}_{4} \mathrm{~F}_{8}$ flow rate is $99 \mathrm{sccm}$, the $\mathrm{APC}$ angle is $81.2^{\circ}$, and the deposition time is $25 \mathrm{~min}$.

The measured polymer sidewall thicknesses [see Fig. 6(a)] for two different structure thicknesses are listed in Table II. The trench aspect ratio for both cases is 10. For each trench, the polymer sidewall thickness near Region I is larger than that near Region II [see Fig. 6(a)]. Typically, the polymer sidewall thickness near Region I is about $30-50 \%$ of the polymer thickness on the top surface of the structure, and the polymer sidewall thickness near Region II is close to that on the trench bottom. Furthermore, the polymer sidewall thickness near Region II decreases as the trench aspect ratio (or trench depth) increases. Also, as the trench aspect ratio (or trench depth) increases, the polymer sidewall deposition rate at Region II decreases. In order to maintain sufficient polymer sidewall thickness near Region II, the polymer deposition time should also be increased as the trench aspect ratio increases, which in turn increases the polymer sidewall thickness near Region I. 


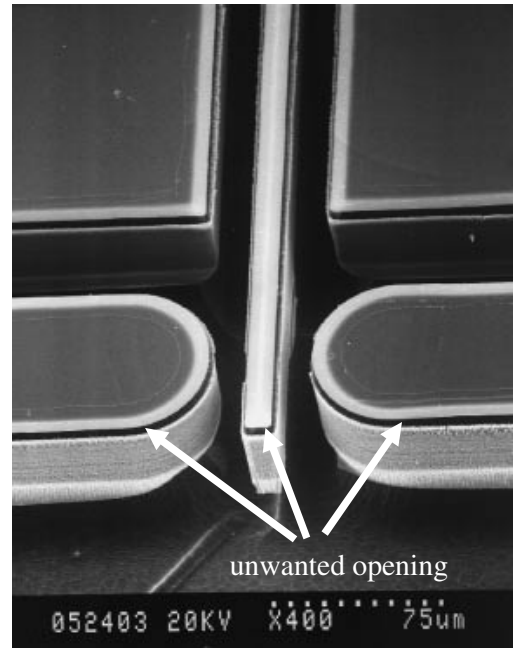

(a)

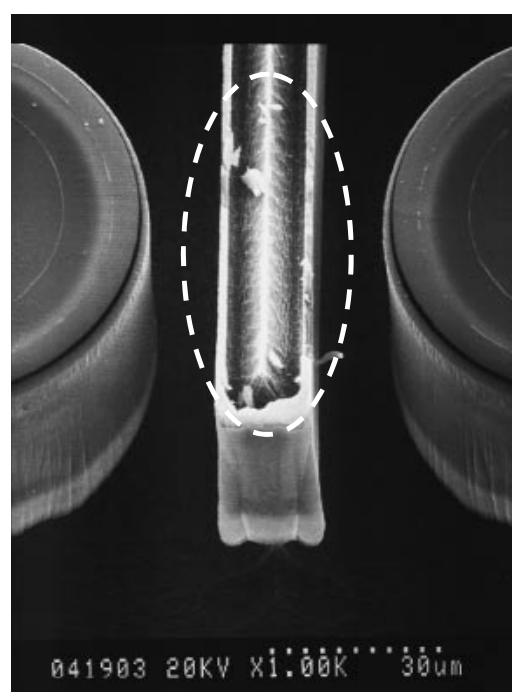

(b)

Fig. 7. (a) Unwanted opening along the top edge of a released structure. (b) Etched cavity (encircled by the dashed line) on top of the structure after the photoresist on top of the structure was removed completely.

\subsection{Floor polymer removal}

This step (Step 3 in previous section) is a combination of physical (ion) bombardment and chemical reaction using fluorine radicals. Only the ion bombardment is dominant in this etching step. $^{22)}$ Therefore, the probability of ion bombardment on the sidewall polymer must be reduced so that the sidewall polymer will not be simultaneously etched. This consideration is similar to the concept of avoiding the RIE lag effect, which is also known as aspect-ratio-dependent etching (ARDE). ${ }^{23,24)}$ Jansen et al. ${ }^{23)}$ proposed that three mechanisms play important roles in the RIE lag effect. These mechanisms are (1) the image force, (2) the IAD, and (3) ion shadowing. Any one of these mechanisms can be controlled by at least one of the four process parameters of the STS ICP-etcher: (1) source coil power, (2) platen power, (3) chamber pressure, and (4) $\mathrm{SF}_{6}$ flow rate. Table I lists the polymer removal times and removal rates for different STS-ICP parameters. The time required for removing the bottom polymer can be reduced by increasing the source power, platen power and SF6 flow rate, or by decreasing the APC angle.

Furthermore, controlling the etching time of floor polymer removal is critical for structure release. The polymer on the trench top [Region I in Fig. 6(a)] is thicker than that on the trench bottom [Region II in Fig. 6(a)], because the polymer deposition rate at the trench top is higher. In order to successfully release structures, the polymer in Region II must be removed without disturbing the protective layer in Region I. However, if the etching time for polymer removal is too long, the protective layer in Region I will be "damaged", as shown in Fig. 6(b). The unwanted opening [see Fig. 6(b)], which is created by the excessive etching of polymer on the top walls (edges) of the trench, will result in undesired etching of the silicon structure during the release process, as shown in Fig. 6(c). Figure 7(a) shows that the structure (silicon) is etched through the unwanted opening, which is along the top edges of the structure, after the structure-releasing step. The photoresist on top of the structure is removed completely if the time for structure release is long enough. For this situation, the schematic and the scanning electron microscopy (SEM) image are shown in Figs. 6(d) and 7(b), respectively.

\subsection{Considerations for structure release}

The structure-releasing step is similar to the BOSCH etching step, except that the platen power is turned off. Therefore, the chemical reaction, which is, in fact, isotropic etching, can be used to release the structure. The releasing time is decided by the width of the structure as well as the lateral etching rate on the trench bottom. In addition, due to the conductance considerations in high-aspect-ratio features $^{20)}$ and the reactant transport effect, ${ }^{21)}$ both the trench opening area and the trench depth affect the flux of etchant species on the trench bottom, and thus affect the lateral etching rate. Figure 8 illustrates that as the trench opening area increases or the trench depth decreases, the lateral

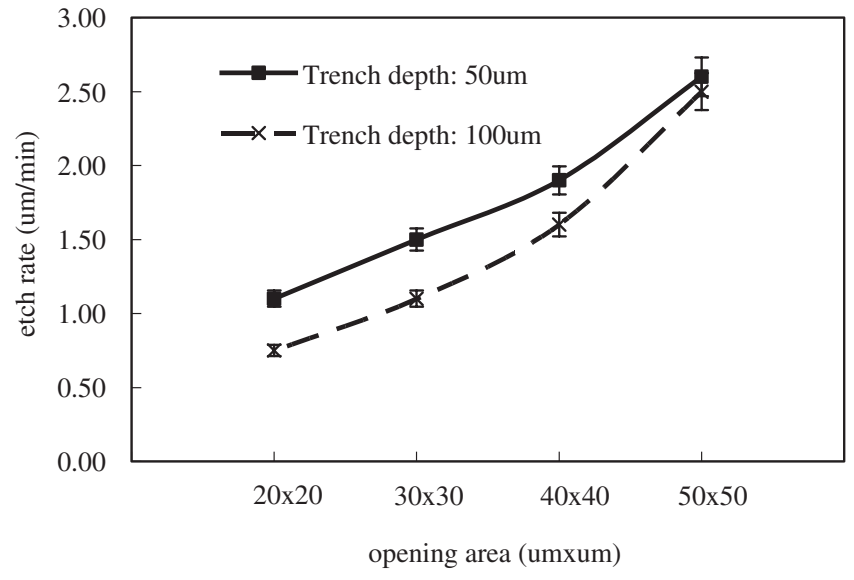

Fig. 8. Lateral etching rate of $\mathrm{SF}_{6}$ for different trench depths and trench dimensions. The process conditions for these curves were as follows: the source coil power was $800 \mathrm{~W}$, the $\mathrm{SF}_{6}$ flow rate was $195 \mathrm{sccm}$, and the APC angle was $81.2^{\circ}$. 
etching rate increases. The process conditions for these curves are as follows: the source coil power is $800 \mathrm{~W}$, the $\mathrm{SF}_{6}$ flow rate is $195 \mathrm{sccm}$, and the APC angle is $81.2^{\circ}$. The lateral etching rate with a trench opening area of $50 \times 50$ $\mu \mathrm{m}^{2}$ is about 2.4-3.4 times that with a $20 \times 20 \mu \mathrm{m}^{2}$ trench area. Moreover, as the trench opening area increases, the difference in lateral etching rates between the two cases (50 $\mu \mathrm{m}$ trench depth and $100 \mu \mathrm{m}$ trench depth) decreases. To release the structure, the structure-releasing time is dominated by the pattern with the smallest trench opening. Therefore, as the trench depth increases, the lateral etching rate decreases, and thus the releasing time must be increased in order to successfully release the structure.

\section{Conclusions}

A process for fabricating high-aspect-ratio structures was presented in this work. This process was extended from the standard $\mathrm{BOSCH}$ process by introducing the steps for protective layer deposition and structure release. All the steps, including trench etching and structure release, can be integrated into a single-run single-mask ICP process, which effectively reduces the process complexity and fabrication cost. By using the proposed process, the fabrication of suspended structures with $120 \mu \mathrm{m}$ thickness was demonstrated. The corresponding aspect ratio of the structure was about 12, and the corresponding aspect ratio of the trench was about 28. The process considerations for each SRM step were clarified. The phenomenon of unwanted openings, which result in undesired etching of the silicon structure during the release step, was also discussed.

\section{Acknowledgement}

This work is supported by the National Science Council, Taiwan, R.O.C. (Contract no. NSC 922212 E 002 084).
1) P. J. Hesketh and D. J. Harrison: Electrochem. Soc. Interface 3 (1994) 21.

2) A. A. Ayon, R. A. Braff, R. Bayt, H. H. Sawin and M. A. Schmidt: J. Electrochem. Soc. 146 (1999) 2730

3) I. W. Rangelow: Surf. Coat. Technol. 97 (1997) 140.

4) F. Laermer and A. Schilp: U.S. Patent 5501893, German Patent DE4241045.

5) M. A. Blauw, T. Zijlstra and E. van der Drift: J. Vac. Sci. Technol. B 19 (2001) 2930.

6) A. A. Ayon, R. Braff, C. C. Lin, H. H. Sawin and M. A. Schmidt: J. Electrochem. Soc. 146 (1999) 339.

7) F. Laermer, A. Schlip, K. Funk and M. Offenberg: Proc. IEEE MEMS, Orlando, FL, 1999, p. 211.

8) F. Laermer and A. Urban: Microelectron. Eng. 67-68 (2003) 349.

9) H.-C. Liu, Y.-H. Lin and W. Hsu: Microsyst. Technol. 10 (2003) 29.

10) K. A. Shaw, Z. L. Zhang and N. C. MacDonald: Sens. Actuators A 40 (1994) 63.

11) M. de Boer, H. Jansen and M. Elwenspoek: Proc. 8th Int. Conf. SolidState Sensors and Actuators (Transducers'95), Stockholm, Sweden, p. 565 .

12) P. F. Indermuehle, C. Linder, J. Brugger, V. P. Jaecklin and N. F. de Rooij: Sens. Actuators A 43 (1994) 346.

13) S. Lee, S. Park and D. Cho: J. Microelectromech. Syst. 8 (1999) 409.

14) Y. B. Gianchandani and K. Najafi: J. Microelectromech. Syst. 1 (1992) 77.

15) J. Hsieh and W. Fang: J. Micromech. Microeng. 12 (2002) 574.

16) A. Bertz, M. Kuechler, R. Knoefler and T. Gessner: Sens. Actuators A 97-98 (2002) 691.

17) E. Sarajlic, M. J. de Boer, H. V. Jansen, N. Arnal, M. Puech, G. Krijnen and M. Elwenspoek: J. Micromech. Microeng. 14 (2004) s70.

18) A. M. Hynes, H. Ashraf, J. K. Bhardwaj, J. Hopkins, I. Johnson and J. N. Shepherd: Sens. Actuators A 74 (1999) 13.

19) S. Lee, S. Park, J. Kim, S. Lee and D. Cho: J. Microelectromech. Syst. 9 (2000) 557.

20) S. Dushman and J. M. Lafferty: Scientific Foundations of Vacuum Technique (Wiley, New York, 1962) 2nd ed., p. 94.

21) J. C. Arnold, D. C. Gary and H. H. Sawin: J. Vac. Sci. Technol. B 11 (1993) 2071.

22) I. W. Rangelow: Vacuum 62 (2001) 279.

23) H. Jansen, M. de Boer, R. Wiegerink, N. Tas, E. Smulders, C. Neagu and M. Elwenspoek: Microelectron. Eng. 35 (1997) 45.

24) M. A. Blauw, T. Zijlstra, R. A. Bakker and E. van der Drift: J. Vac. Sci. Technol. B 18 (2000) 3453. 